\title{
Management of Defects on Lower Extremities with the Use of Matriderm and Skin Graft
}

\author{
Jun-Young Choi, Seong-Hun Kim, Gwang-Jin Oh, Si-Gyun Roh, Nae-Ho Lee, Kyung-Moo Yang \\ Department of Plastic and Reconstructive Surgery, Chonbuk National University Medical School, Jeonju, Korea
}

Background The reconstruction of large skin and soft tissue defects on the lower extremities is challenging. The skin graft is a simple and frequently used method for covering a skin defect. However, poor skin quality and architecture are well-known problems that lead to scar contracture. The collagen-elastin matrix, Matriderm, has been used to improve the quality of skin grafts; however, no statistical and objective review of the results has been reported.

Methods Thirty-four patients (23 male and 11 female) who previously received a skin graft and simultaneous application of Matriderm between January 2010 and June 2012 were included in this study. The quality of the skin graft was evaluated using Cutometer, occasionally accompanied by pathologic findings.

Results All 34 patients showed good skin quality compared to a traditional skin graft and were satisfied with their results. The statistical data for the measurement of the mechanical properties of the skin were similar to those for normal skin. In addition, there was no change in the engraftment rate.

Conclusions The biggest problem of a traditional skin graft is scar contracture. However, the dermal matrix presents an improvement in skin quality with elastin and collagen. Therefore, a skin graft along with a simultaneous application of Matriderm is safe and effective and leads to a significantly better outcome from the perspective of skin elasticity.

Keywords Lower extremity / Elasticity / Skin transplantation
Correspondence: Si-Gyun Roh Department of Plastic and Reconstructive Surgery, Chonbuk National University Hospital, Chonbuk National University Medical School, 20 Geonji-ro, Deokjin-gu, Jeonju 561-712, Korea Tel: +82-63-250-1860 Fax: +82-63-250-1866 E-mail: pssroh@jbnu.ac.kr

No potential conflict of interest relevant to this article was reported.

\section{INTRODUCTION}

The reconstruction of damaged skin and soft tissue in the lower extremities is a challenging problem for plastic surgeons. The lower extremities have limited surrounding soft tissues for the reconstruction of the damaged area. In addition, functional or cosmetic issues are a frequent occurrence in areas near the knees with a large range of motion upon completion of the reconstruction. Moreover, if the damage of the lower extremity is wide, both a free flap and a local flap have limitations with re- spect to the range of reconstruction. Therefore, a split-thickness skin graft is frequently used. The split-thickness skin graft has minor issues such as those related to skin elasticity, and an unsatisfactory cosmetic outcome; as a result, a complication known as scar contracture is a common occurrence [1].

With respect to damage that has occurred in the lower extremities, it is important to find a method that takes into account not only the primary goal of reconstruction but also the appropriate cosmetic perspective $[2,3]$. Various artificial dermal substitutes have been developed in order to minimize scar contracture, 
which is a disadvantage of the split-thickness skin graft. Among them, the effectiveness of Integra (Integra Life Science Corporation, Plainsboro, NJ, USA) has been proven in various clinical trials $[4,5]$. However, Integra has a disadvantage, in that 2 to 3 weeks after the initial application to the damaged area, a second surgical treatment step is necessary for the removal of the uppermost layer made of silicone [6].

Matriderm (Dr. Otto Suwelack Skin \& Health Care AG, Billerbeck, Germany), a three-dimensional structure, is a matrix containing I, II, and V-type collagen extracted from bovine ligament and dermis, and alpha-elastin hydrolysates. It can be applied concurrently with the split-thickness skin graft for the reconstruction of the lower extremities; thus, secondary surgery is not required $[7,8]$. In addition, the following can be minimized by using Matriderm: reduction of skin elasticity and flexibility (after completion of the split-thickness skin graft without any other procedure), differences in skin color and texture, and functional problems due to scar contracture. However, because no objective assessment on this subject has been reported in the literature, this study was conducted to demonstrate usefulness of matriderm.

\section{METHODS}

This study was conducted at our institution from January 2010 to June 2012. A total of 34 patients (male, 23; female, 11; mean age, 48 years; age distribution, 10-77 years) who received an application of Matriderm and a split-thickness skin graft for skin and soft tissue defects on the lower extremities and buttocks were included. In addition, retrospective investigations were conducted by a review of medical records and assessment of skin elasticity using Cutometer (Courage \& Khazaka Electronics, Köln, Germany), photos, and pathology. With the application of general anesthesia, all 34 patients included in the study received Matriderm on the damaged area of the lower extremities; the split-thickness skin graft was performed concurrently. The graft was fixed with sutures and a stapler, and a tie-over dressing was used to promote engraftment and to prevent hematoma. To stabilize the wound, the lower extremities were fixed in order to prevent movement by using a splint for 7 to 14 days. Patients were treated in a hospital as inpatients until sufficient recovery was achieved. Due to issues regarding cost, not all of the damaged areas could be covered with Matriderm in two patients, and only the split-thickness skin graft was performed on some areas.

All patients underwent follow-up for at least 6 months, and photographs were taken in the second and the sixth month after the procedure. Skin elasticity was measured in the sixth month using the Cutometer. One patient underwent biopsy in two areas. One area had Matriderm and the split-thickness skin graft (knee) applied concurrently, and the other area (lower leg) had only the split-thickness skin graft. In addition, such a biopsy was performed in the sixth month for measurement of the elastin density of two areas from the perspective of pathology.

Cutometer is a commercial device used for an objective assessment of skin elasticity, and the investigating ruler has a 6-mm round inlet port at the center. During measurement, the skin is sucked into the inlet port of the investigating ruler and returned to its previous shape by passing through the inlet port when negative pressure has dissipated, and the skin depth is measured at this moment using light. In addition, a graph of the depth of skin sucked in per unit time is generated. While absolute values

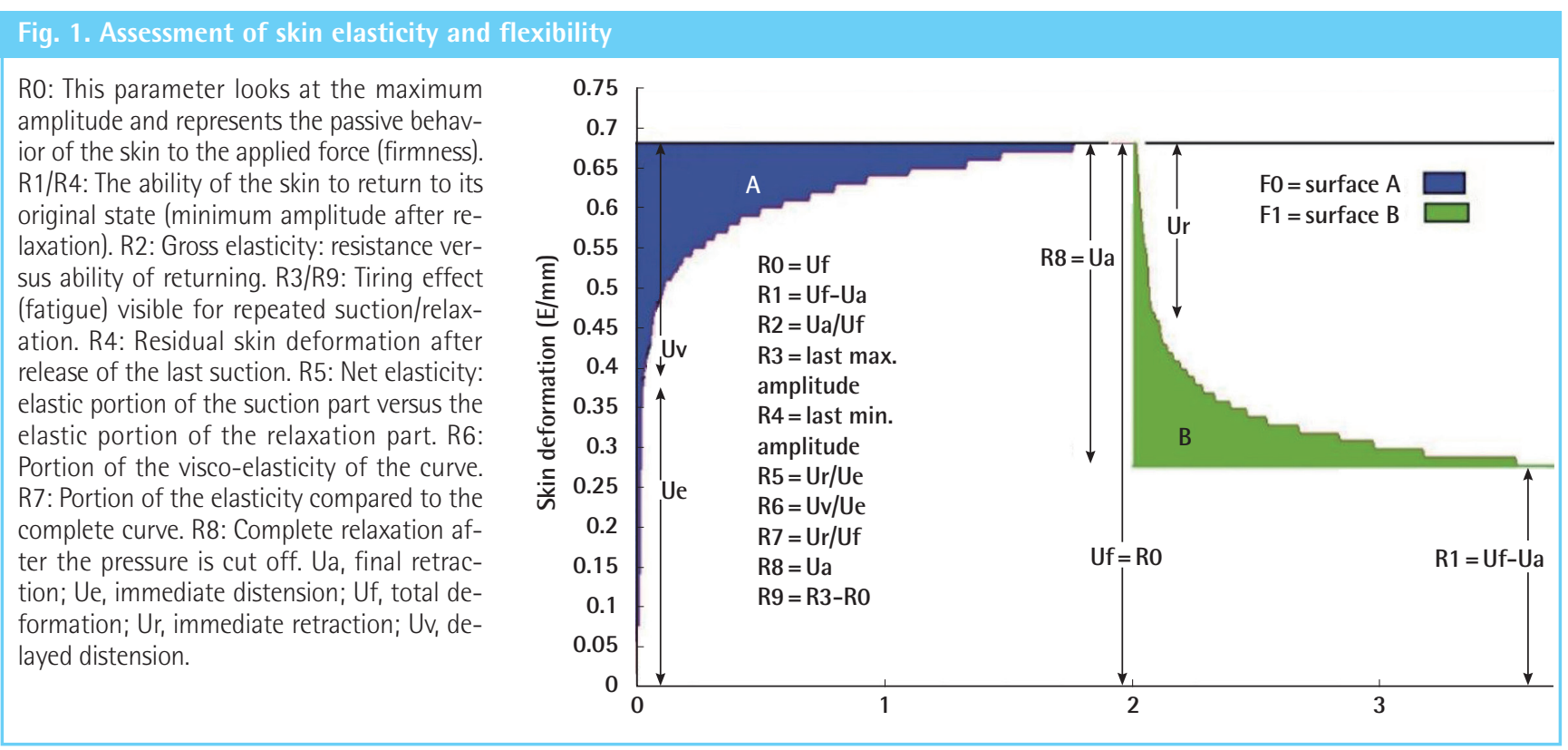


are determined according to the skin depth, their ratios change depending on the characteristics of the skin areas irrespective of the skin depth. Therefore, the obtained values can be considered objective skin elasticity measurement values (Fig. 1) [9].

\section{RESULTS}

A total of 34 patients received treatment as originally planned, and there was no case of follow-up failure based on death or change of address of the patient during the six-month follow-up period. The causes of damage to the lower extremities varied, including burns, open fractures, crushing injuries, congenital melanocytic nevus, malignant skin tumors, and flap donor sites. The engraftment rate of skin grafts using Matriderm was $97 \%$ at our institution.

The elasticity and flexibility of the graft were compared with those of the surrounding normal skin tissues using Cutometer. Each value was investigated by dividing it as follows: $\mathrm{Ur} / \mathrm{Ue}$ (elastic function), Ur/Uf (gross elasticity), Ua/Uf (biological elasticity), and Uv/Ue (viscoelastic ratio) (Table 1). The measured values showed statistically similar elasticity and flexibility as compared to the surrounding normal skin and values obtained in an independent t-test (Table 2), and demonstrated improvement of skin quality as compared to that in the case of an existing simple split-thickness skin graft. During scar revision, biopsy was performed at the graft site of one patient at the sixth month. Upon completion of the Verhoeff van Gieson stain, the area with Matriderm and the split-thickness skin graft (anterior thigh and lateral knee area) had a higher elastin density than the area (calf) that had only the split-thickness skin graft (Fig. 2). Further, a thicker dermis layer was observed by a pathologist in the Verhoeff van Gieson stain slide.

\section{Case study 1}

This patient was a 40-year-old female patient, who was admitted through the emergency room with multiple open fractures and a crushing injury of the soft tissue in the right lower extremity resulting from an automobile accident. The patient had necrosis in the right femoral region, around the knee joint, on the fullthickness skin tissues of the popliteal fossa, and in the posterior region of the leg. An external fixator procedure for a fracture was performed in the orthopedics department on the second day of the patient's hospital stay, and the necrotic tissue was removed in the plastic surgery department on the 14th day of her hospital stay. On the 25th day of her hospital stay, Matriderm was applied and the split-thickness skin graft procedure was performed on the anterior thigh, around the knee joint, and on the lower leg; the elasticity and flexibility test and a photo shoot of the skin were performed in the sixth month after the skin graft. From the follow-up pictures, we found that the knee joint

\section{Table 1. Data of 20 patients}

\begin{tabular}{|c|c|c|c|c|c|c|}
\hline \multirow{2}{*}{ Patient no. } & \multirow{2}{*}{ Age $(y r) / S e x$} & \multirow{2}{*}{ Graft region } & \multicolumn{2}{|c|}{ Ur/Ue } & \multicolumn{2}{|c|}{ Ur/Uf } \\
\hline & & & Graft & Normal & Graft & Normal \\
\hline 1 & $28 / F$ & Knee & 0.998 & 1.025 & 0.612 & 0.654 \\
\hline 2 & $68 / M$ & Thigh & 1.018 & 1.032 & 0.624 & 0.658 \\
\hline 3 & $47 / F$ & Ankle & 1.033 & 1.054 & 0.633 & 0.667 \\
\hline 4 & $34 / M$ & Calf & 1.044 & 1.055 & 0.613 & 0.641 \\
\hline 5 & $49 / M$ & Lower leg & 1.011 & 1.062 & 0.624 & 0.638 \\
\hline 6 & $60 / F$ & Thigh & 1.024 & 1.044 & 0.598 & 0.644 \\
\hline 7 & $20 / F$ & Calf & 1.011 & 1.032 & 0.635 & 0.653 \\
\hline 8 & $76 / M$ & Thigh & 1.014 & 1.045 & 0.603 & 0.655 \\
\hline 9 & $39 / M$ & Calf & 1.018 & 1.038 & 0.621 & 0.674 \\
\hline 10 & $57 / M$ & Thigh & 1.047 & 1.047 & 0.601 & 0.623 \\
\hline 11 & $10 / F$ & Ankle & 1.033 & 1.042 & 0.622 & 0.669 \\
\hline 12 & $77 / M$ & Thigh & 1.025 & 1.049 & 0.613 & 0.655 \\
\hline 13 & $57 / F$ & Knee & 1.001 & 1.058 & 0.612 & 0.678 \\
\hline 14 & $22 / M$ & Thigh & 1.033 & 1.063 & 0.622 & 0.644 \\
\hline 15 & $44 / M$ & Knee & 1.031 & 1.048 & 0.627 & 0.677 \\
\hline 16 & $65 / F$ & Lower leg & 1.027 & 1.036 & 0.611 & 0.674 \\
\hline 17 & $61 / M$ & Calf & 1.024 & 1.057 & 0.615 & 0.665 \\
\hline 18 & $55 / F$ & Thigh & 1.011 & 1.048 & 0.608 & 0.647 \\
\hline 19 & $41 / M$ & Knee & 1.012 & 1.059 & 0.601 & 0.635 \\
\hline 20 & $50 / M$ & Knee & 1.018 & 1.049 & 0.621 & 0.648 \\
\hline
\end{tabular}

Ur/Ue and Ur/Uf values are compared with those of the surrounding normal skin tissues by using the Cutometer. Ur, immediate retraction; Ue, immediate distension; Uf, total deformation. 
Table 2. Statistical significance between grafted skin and surrounding skin

\begin{tabular}{|c|c|c|c|c|c|c|c|c|c|}
\hline \multirow{3}{*}{ Value } & \multicolumn{2}{|c|}{$\begin{array}{l}\text { Levene's test for } \\
\text { equality of variances }\end{array}$} & \multicolumn{7}{|c|}{ T-test for equality of means } \\
\hline & \multirow{2}{*}{$\mathrm{F}$} & \multirow{2}{*}{ Sig. } & \multirow[t]{2}{*}{$\mathrm{T}$} & \multirow{2}{*}{ df } & \multirow{2}{*}{$\begin{array}{c}\text { Sig. } \\
\text { (2-tailed) }\end{array}$} & \multirow{2}{*}{$\begin{array}{c}\text { Mean } \\
\text { difference }\end{array}$} & \multirow{2}{*}{$\begin{array}{l}\text { Std. error } \\
\text { difference }\end{array}$} & \multicolumn{2}{|c|}{$\begin{array}{l}95 \% \text { Confidence } \\
\text { interval of the difference }\end{array}$} \\
\hline & & & & & & & & Lower & Upper \\
\hline \multicolumn{10}{|l|}{ Ur/Ue } \\
\hline Equal variances assumed & 0.969 & 0.331 & -0.3012 & 38 & 0.178 & -0.02550 & 0.00374 & -0.3308 & -0.1792 \\
\hline Equal not variances assumed & - & - & -0.3012 & 36.600 & 0.178 & -0.02550 & 0.00374 & -0.3309 & -0.1791 \\
\hline \multicolumn{10}{|l|}{ Ur/Uf } \\
\hline Equal variances assumed & 2.365 & 0.132 & -0.6425 & 38 & 0.065 & -0.03915 & 0.00415 & -0.6756 & -0.3074 \\
\hline Equal not variances t assumed & - & - & -0.6425 & 33.674 & 0.065 & -0.03915 & 0.00415 & -0.6759 & -0.3071 \\
\hline
\end{tabular}

There was no significant difference in the Ur/Ue values ( $\mathrm{t}[38]=-0.3012, \mathrm{P}=0.178$ ) and the Ur/Uf values ( $\mathrm{t}[38]=-0.6425, \mathrm{P}=0.065)$, according to an independent $\mathrm{t}$-test. F, F-test; Sig., significance probability'; df, degree of freedom; Std, standard.

\section{Fig. 2. The comparison of elastin density}

(A) Pathological findings of the area on which Matriderm was applied and the split-thickness skin graft was performed concurrently. In addition, the elastin dyed in pink has high density and the new dermis is thick (Verhoeff van Gieson stain, $\times 200$ ). (B) Pathological findings of the area that had only the split-thickness skin graft. Here, the elastin dyed in pink has low density, and the new dermis is thinner (Verhoeff van Gieson stain, $\times 200$ ).

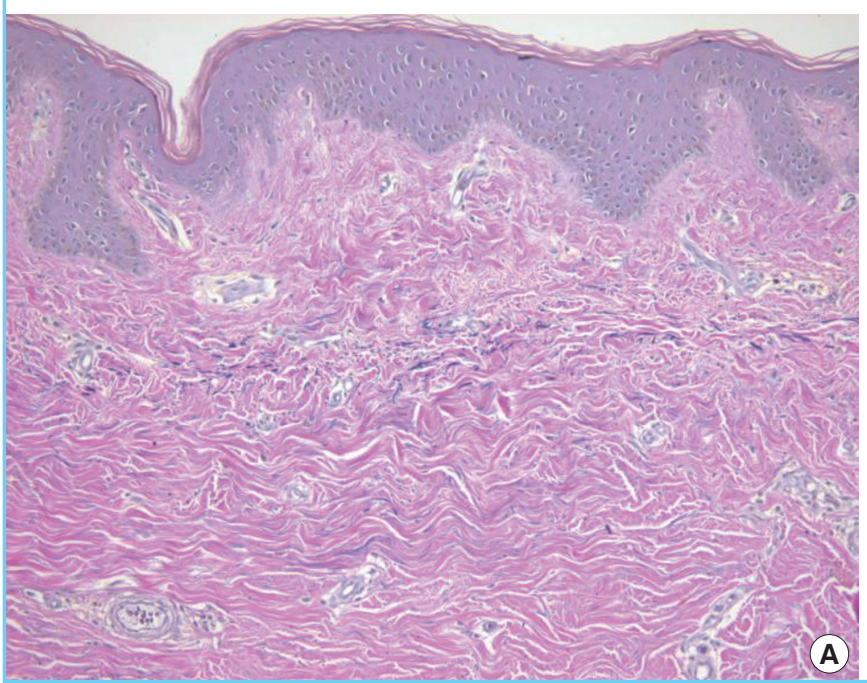

showed a normal range of motion (knee flexion, $0^{\circ}-120^{\circ}$ ), and scar contracture was not observed (Fig. 3).

\section{Case study 2}

This patient was a 17-year-old male. An external fixator operation was performed in the orthopedics department for an open fracture of the right fibula and tibia resulting from an automobile accident. The patient was then sent to the plastic surgery department, where he underwent the removal of necrotic tissue on the eighth day of his hospital stay; the consequential soft tissue damage was treated using the anterolateral thigh free flap on the 15th day of his hospital stay. In addition, Matriderm was applied, and the split-thickness skin graft procedure was performed in the lateral lower leg region on the 29th day of the patient's hospital stay. The skin elasticity and flexibility test and the

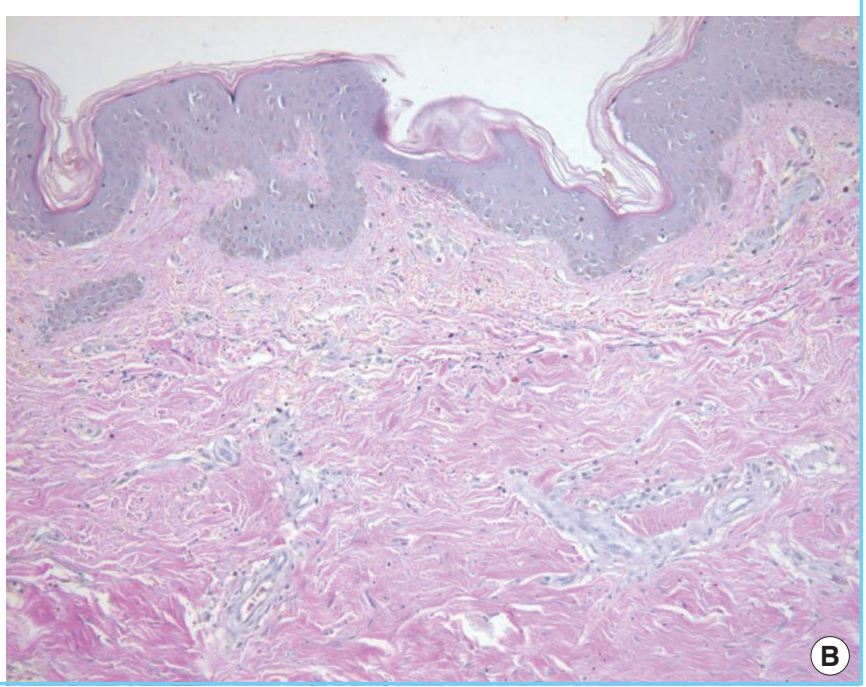

photo shoot were performed in the sixth month after surgery, and no hypertrophic scar or scar contracture was observed in the grafted area (Fig. 4).

\section{DISCUSSION}

Damaged skin and soft tissue in the lower extremities is a challenging problem for plastic surgeons. As a method for the reconstruction of the damaged area, flap surgery provides excellent coverage for the soft tissue. However, it requires multiple operations and is potentially problematic from a cosmetic perspective because it is excessively thick. Furthermore, if the damage is wide, it is impossible to cover the damaged area with only flap surgery. For the aforementioned reasons, flap surgery is not considered the absolute first choice for lower extremity reconstruc- 


\section{Fig. 3. Case study 1:40-year-old female}

(A) Traumatic soft tissue necrosis findings on the right lower extremity. (B) Matriderm was applied and the split-thickness skin graft procedure was performed on the 25th day of the patient's hospital stay. (C) A photograph of the sixth-month follow-up after the transplant surgery; note that here, scar contracture is absent.
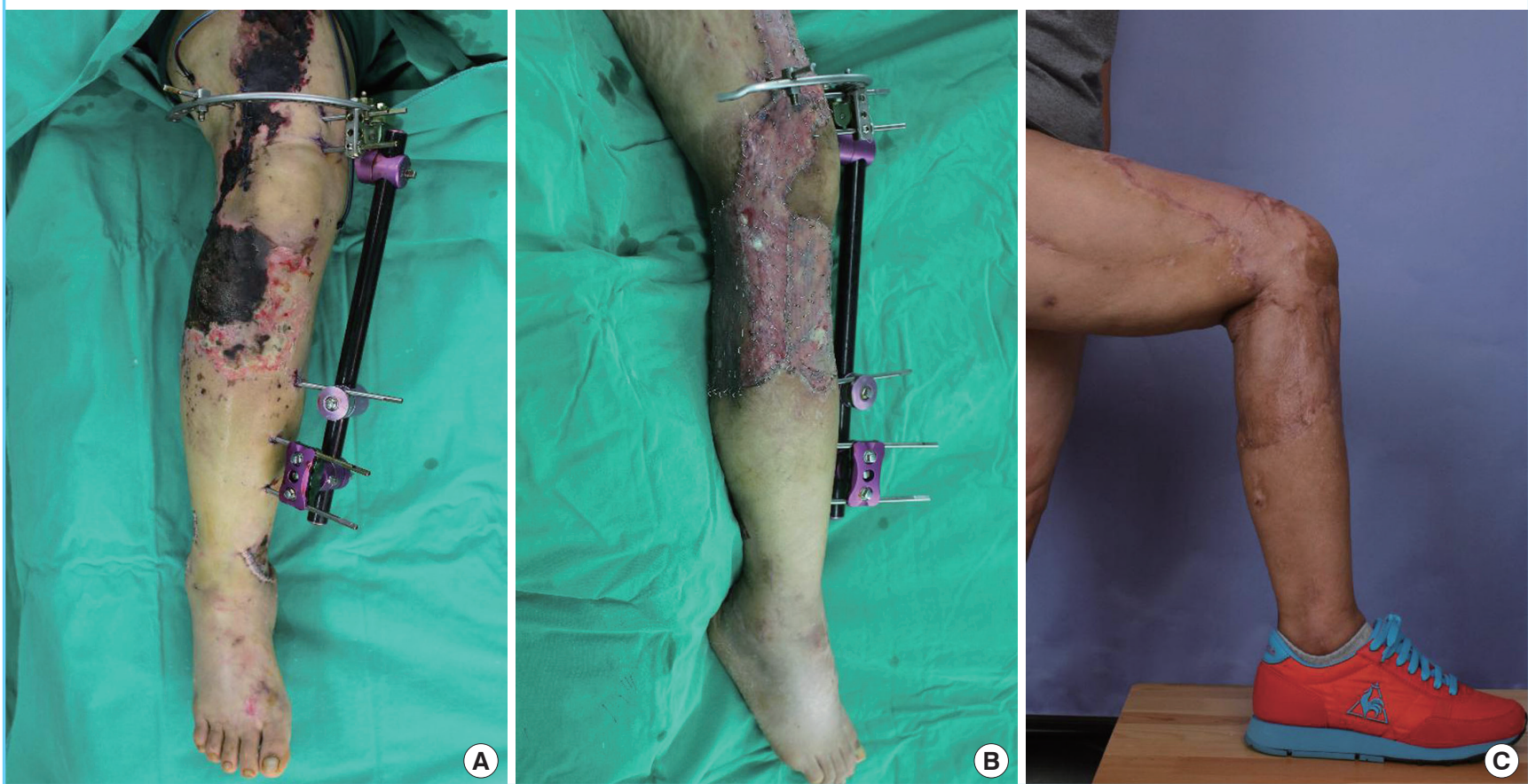

Fig. 4. Case study 2: 15-year-old male

(A) The bone is exposed due to an open fracture of the right lower leg, and wide soft tissue damage is shown. (B) A free flap procedure was performed on the anterolateral thigh on the 15th day of the patient's hospital stay. Accordingly, Matriderm was applied and the split-thickness skin graft procedure was performed for the remaining damaged area on the 29th day of the patient's hospital stay. (C) Hyperplastic scar and scar contracture are absent in the sixth month after the surgery.
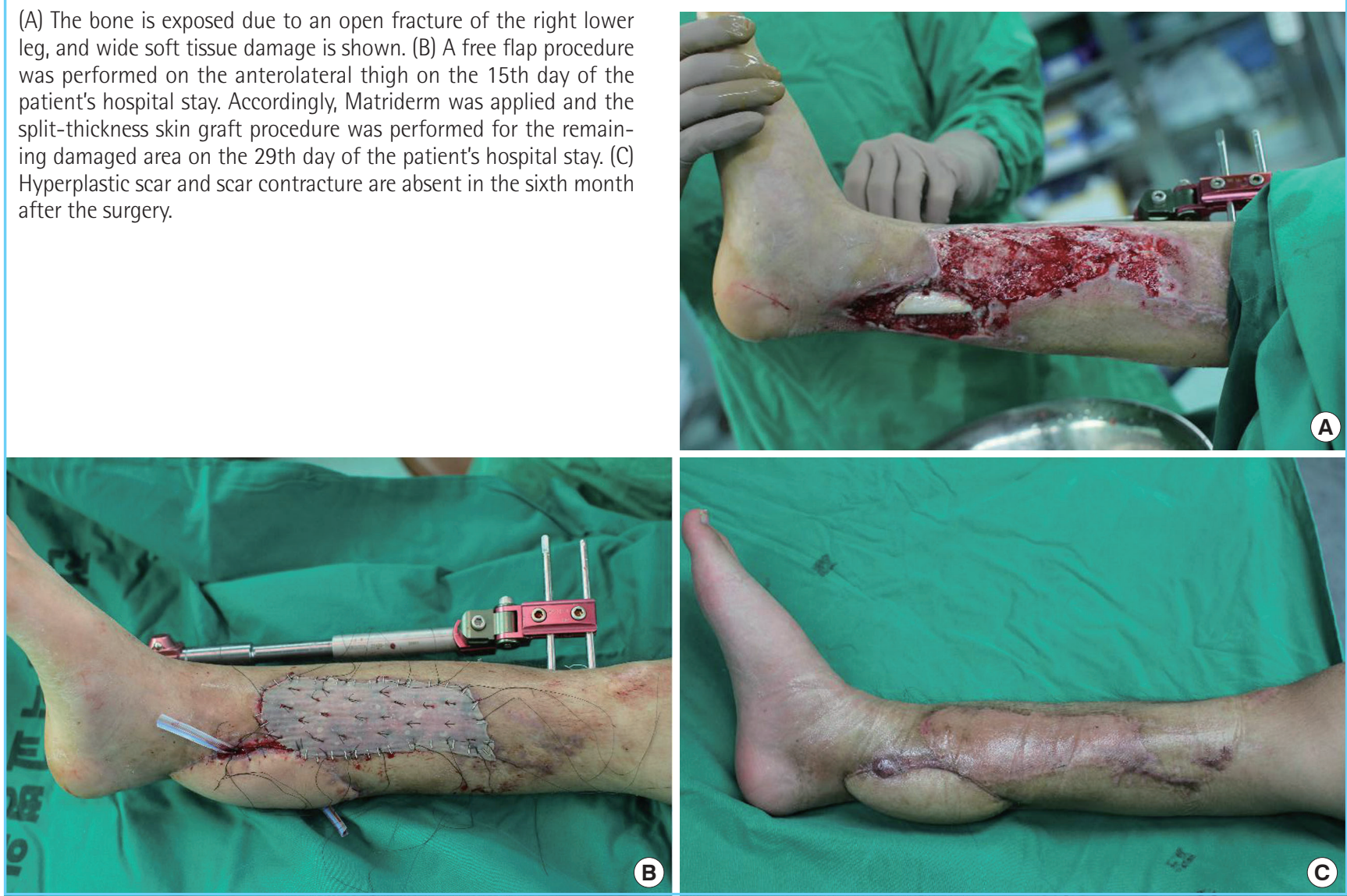
tion. Many studies have recently reported that an artificial dermal substitute increases the quality of a skin graft and offers a relatively easy reconstruction method $[10,11]$.

From the present study, it was confirmed that the reconstruction of skin and soft tissue defects in the lower extremities is possible with only a single step of surgical treatment without engraftment failure. This can be a major advantage when compared with other methods, which are time consuming and require multiple steps of surgical treatment. Eventually, stabilization of the surgical wound becomes more rapid. Therefore, being able to exercise at an earlier time and an earlier return to normal daily activities is possible. This can eventually lead to a reduction in healthcare costs.

Matriderm, which was made commercially available in 2004, primarily minimizes scar contracture. Second, it increases skin elasticity and flexibility. This was proved by an analysis using the Cutometer, which is designed to measure the elasticity of the upper skin layer using negative pressure that mechanically deforms the skin. Therefore, Matriderm compensates for the disadvantage of the existing split-thickness skin graft. Although thus far, study results related to such advantages have rarely been reported in the literature, these advantages were objectively demonstrated in this study.

However, this study has certain limitations: There was no comparison group of patients who underwent only split-thickness skin graft surgery. In case 1, biopsy was performed only in the calf region and only on the graft to which Matriderm could not be topically applied. In addition, skin elasticity or flexibility could not be measured because the range was too narrow. Because the follow-up period was less than 1 year, a long term follow-up study will be necessary in the future.

In conclusion, the goal of this study was to objectively confirm the usefulness and advantages of Matriderm, an artificial dermal substitute, and to demonstrate that the previously proposed theory of substitutes playing a negative role in the delivery of the necessary blood and nutrients for the engraftment of a graft is unfounded. The average engraftment rate of skin grafts using Matriderm was $96 \%$. There was no occurrence of a lowered engraftment rate due to the widened transport distance of nutrients and oxygen into the graft because of the artificial dermal substitute inserted in the middle $[12,13]$. We believe that the concurrent use of Matriderm and the split-thickness skin graft can not only bring about functional and cosmetic improvement but also play the role of a temporary barrier with the following advantages: a hemostatic effect, reduction of scar contracture, prevention of infections, maintenance of the elasticity and flexibility of the existing skin, and reduction of the scar tissue. In this study, good results were obtained by concurrently using Matri- derm and the split-thickness skin graft for the damaged area in the lower extremities.

\section{REFERENCES}

1. Haslik W, Kamolz LP, Manna F, et al. Management of fullthickness skin defects in the hand and wrist region: first long-term experiences with the dermal matrix Matriderm. J Plast Reconstr Aesthet Surg 2010;63:360-4.

2. Yannas IV, Burke JF. Design of an artificial skin. I. Basic design principles. J Biomed Mater Res 1980;14:65-81.

3. Lamme EN, de Vries HJ, van Veen H, et al. Extracellular matrix characterization during healing of full-thickness wounds treated with a collagen/elastin dermal substitute shows improved skin regeneration in pigs. J Histochem Cytochem 1996;44:1311-22.

4. Hansbrough JF, Cooper ML, Cohen R, et al. Evaluation of a biodegradable matrix containing cultured human fibroblasts as a dermal replacement beneath meshed skin grafts on athymic mice. Surgery 1992;111:438-46.

5. King WW, Lam PK, Liew CT, et al. Evaluation of artificial skin (Integra) in a rodent model. Burns 1997;23 Suppl 1: S30-2.

6. Burke JF, Yannas IV, Quinby WC Jr, et al. Successful use of a physiologically acceptable artificial skin in the treatment of extensive burn injury. Ann Surg 1981;194:413-28.

7. Middelkoop E, de Vries HJ, Ruuls L, et al. Adherence, proliferation and collagen turnover by human fibroblasts seeded into different types of collagen sponges. Cell Tissue Res 1995;280:447-53.

8. de Vries HJ, Middelkoop E, Mekkes JR, et al. Dermal regeneration in native non-cross-linked collagen sponges with different extracellular matrix molecules. Wound Repair Regen 1994;2:37-47.

9. Barel AO, Courage W, Clarys P, et al. Suction method for measurement of skin mechanical properties: the cutometer. In: Serup J, Jemec GB, editros. Handbook of noninvasive methods and the skin. Boca Raton: CRC Press;1995. p.33540.

10. van Zuijlen PP, van Trier AJ, Vloemans JF, et al. Graft survival and effectiveness of dermal substitution in burns and reconstructive surgery in a one-stage grafting model. Plast Reconstr Surg 2000;106:615-23.

11. Wainwright D, Madden M, Luterman A, et al. Clinical evaluation of an acellular allograft dermal matrix in full-thickness burns. J Burn Care Rehabil 1996;17:124-36.

12. Hansbrough JF, Dore C, Hansbrough WB. Clinical trials of a living dermal tissue replacement placed beneath meshed, 
split-thickness skin grafts on excised burn wounds. J Burn Care Rehabil 1992;13:519-29.

13. Ryssel H, Gazyakan E, Germann G, et al. The use of Matri-
Derm in early excision and simultaneous autologous skin grafting in burns: a pilot study. Burns 2008;34:93-7. 